\title{
Calidad de vida de personas con discapacidad intelectual en centros de formación laboral*
}

\section{Quality of Life of People with Intellectual Disabilities at Vocational Training Schools Qualidade de vida de pessoas com deficiência intelectual em centros de formação laboral}

\author{
Lilian Castro Durán, ${ }^{* *}$ Gamal Cerda Etchepare, ${ }^{* *}$ Viviana Vallejos Garcías, ${ }^{* *}$ \\ Daniela Zúñiga Vásquez, ${ }^{* *}$ Rufino Cano González ${ }^{* * *}$ \\ ${ }^{* *}$ Universidad de Concepción, Chile. ${ }^{* * *}$ Universidad de Valladolid, España.
}

Doi: dx.doi.org/10.12804/ap134.1.2016.12

\section{Resumen}

Esta investigación presenta un perfil comparativo de calidad de vida de personas con discapacidad intelectual que asisten a centros de formación laboral. Se aplicó la Escala Integral, Evaluación Objetiva y Subjetiva de Calidad de Vida a personas con discapacidad intelectual ( $N=751$ para la evaluación subjetiva) y a los profesionales a cargo ( $N=82$ para la evaluación objetiva). La autopercepción de las personas respecto de su calidad de vida es superior a la percepción de los profesionales a su cargo y destaca la dimensión de bienestar laboral; y en la evaluación objetiva, la dimensión inclusión social.
En ambas evaluaciones, la autodeterminación es una de las dimensiones con menor nivel de consecución. En la evaluación subjetiva, el grupo femenino tiene una mejor percepción en la dimensión bienestar laboral, y el grupo masculino en la dimensión bienestar emocional y físico. En la evaluación objetiva existen diferencias significativas a favor del grupo masculino en inclusión social, y en bienestar laboral a favor del grupo femenino. Los profesionales del paradigma social puntúan más alto en las dimensiones de autodeterminación, bienestar material e inclusión social.

Palabras clave: calidad de vida; discapacidad; entrenamiento; género.

* Esta investigación fue financiada por la Comisión Nacional de Investigación Científica y Tecnológica (Conicyt) de Chile. Apoyo Convenio de desempeño UCO 1203. Proyecto Fondecyt de Iniciación n. ${ }^{\circ} 11121216$ : Calidad de vida de jóvenes con discapacidad intelectual y su relación con la formación laboral.

** Lilian Castro Durán, jefe de Carrera Educación Diferencial, Departamento Curriculum, Facultad de Educación, Universidad de Concepción, Chile; Gamal Cerda Etchepare, profesor asociado del Departamento de Metodología de la Investigación e Informática Educacional, de la Facultad de Educación de la Universidad de Concepción; Viviana Vallejos Garcías, profesora de la carrera Educación Diferencial, Departamento Curriculum, Facultad de Educación, Universidad de Concepción; Daniela Zúñiga Vásquez, profesora de Educación Diferencial en el Colegio Diferencial Per Se, Concepción.

*** Rufino Cano González, profesor titular, Facultad de Educación y Trabajo Social Departamento de Pedagogía, Área de Métodos de Investigación y Diagnóstico en Educación de la Universidad de Valladolid, España.

La correspondencia relacionada con este artículo debe ser dirigida a Lilian Castro Durán, Facultad de Educación, Universidad de Concepción, Casilla 160, Concepción, Chile. Correo electrónico: lilicastro@udec.cl

Cómo citar este artículo: Castro, L., Cerda, G., Vallejos, V., Zúñiga, D. \& Cano, R. (2016). Calidad de vida de personas con discapacidad intelectual en centros de formación laboral. Avances en Psicología Latinoamericana, 34(1), 175-186. doi: dx.doi.org/10.12804/apl34.1.2016.12 


\section{fibstract}

This study reports a comparative profile of Quality of Life of people with intellectual disabilities attending vocational training schools in Chile. To this end, the QOL profile of people with disabilities $(N=751)$ was obtained by means of a Subjective Assessment. Equally, the professionals in charge of the people with intellectual disabilities $(N=82)$ were also assessed by means of an Objective Assessment. The self-perception of people with intellectual disabilities in respect to their Quality of Life was higher than the perception of the professionals in charge of them. In the Subjective Assesment used to obtain the profile of people with disabilities, the Occupational Wellbeing dimension stood out, and in the Objective Assessment, the Social Inclusion dimension emerged. Furthermore, Self-determination stood out as one of the dimensions with the lowest level of achievement in both assessments. It was also found that the female group had a better perception of the Occupational Wellbeing dimension and the male group had a better perception of the Emotional and Psychological Wellbeing dimension. In respect to the Objective Assessment, significant differences in the Social Inclusion dimension were found, favoring the male group; likewise, significant differences favoring the female group were also found regarding the Occupational Wellbeing dimension. Additionally, the highest scores in the dimensions of Self-determination, Material Wellbeing and Social Inclusion were given by the professionals working at schools with a social paradigm.

Keywords: quality of life; disabilities; training; gender.

\section{Resumo}

A pesquisa apresenta um perfil comparativo de qualidade de vida de pessoas com deficiência intelectual, que assistem a centros de formação laboral. Aplicou-se a Escala Integral, Avaliação Objetiva e Subjetiva de Qualidade de Vida a pessoas com deficiência intelectual $(N=751)$, Avaliação Subjetiva, e aos profissionais a cargo $(N=82)$, Avaliação Objetiva. A autopercepção das pessoas com respeito a sua qualidade de vida é superior à percepção dos profissionais a seu cargo, destacando a dimensão de Bem-estar Laboral; e na Avaliação Objetiva a dimensão Inclusão Social. Em ambas as avaliações, a Autodeterminação é uma das dimensões com menor nível de consecução. Na Avaliação Subjetiva, o grupo feminino tem uma maior percepção na dimensão Bem-estar Laboral, e o grupo masculino na dimensão Bem-estar Emcional e Físico. Na Avaliação Objetiva existem diferenças significativas a favor do grupo masculino em Inclusão Social e em Bem-estar Laboral a favor do grupo feminino. Os profissionais do paradigma social pontuam mais alto nas dimensões de Autodeterminação, Bem-estar material e Inclusão social.

Palavras-chave: qualidade de vida; deficiência; formação; gênero.

El mejoramiento de la calidad de vida de las personas se ha constituido paulatinamente en una finalidad mundial, pues ello repercute en la percepción de bienestar general de las personas, independiente de su condición, nivel socioeconómico, raza o cultura. Por lo mismo, resulta natural que este constructo se haya extendido al ámbito de las personas con discapacidad intelectual, lo que, a su vez, ha redundado en la necesidad de contar con mecanismos e instrumentos de evaluación pertinentes y válidos para su examen (Schalock \& Verdugo, 2002/2003; Verdugo \& Schalock, 2009). Si bien existen diversos modelos de calidad de vida, el modelo al cual se ha adherido la presente investigación es el propuesto por Schalock y Verdugo, que es el de mayor aprobación en el ámbito internacional. Diversas investigaciones sostienen que este modelo multidimensional de la calidad de vida presenta una clara consistencia estructural en las ocho dimensiones que lo componen (Gómez, Verdugo, Arias \& Arias, 2010), y muestra índices robustos de carácter estadístico necesarios para considerarlo un referente teórico con base científica (Wang, Schalock, Verdugo \& Jenaro, 2010). Desde esta perspectiva, Schalock y Verdugo (2002/2003) plantean el constructo de calidad de vida como 
"un estado deseado de bienestar personal que es multidimensional; tiene propiedades éticas —universales y émicas - ligadas a la cultura; tiene componentes objetivos y subjetivos; y está influenciada por factores personales y ambientales" (Verdugo, Gómez, Arias \& Schalock, 2009, p. 10).

Este constructo es consistente con lo planteado en las últimas dos décadas, referido al modelo multidimensional y ecológico y a la necesidad de incorporar indicadores objetivos y subjetivos en su medición (Gómez, Verdugo \& Arias, 2010; Wang et al., 2010); de ahí que estos están "estrechamente ligados al contexto educativo, sanitario, habilitador o rehabilitador en que las personas viven y se relacionan con los demás" (Henao y Gil, 2009, p. 121). Las ocho dimensiones propuestas por el modelo son: bienestar emocional, bienestar material, bienestar físico, relaciones interpersonales, desarrollo personal, autodeterminación, inclusión social y derechos.

En el contexto educativo, por ejemplo, las ocho dimensiones del modelo, además se examinan a la luz del contexto donde se desenvuelve la persona, el tipo de educación que recibe (regular/especial), su salud (física/mental/psíquica) o nivel intelectual, ya que todos estos elementos deben analizarse respecto de su posible impacto en la calidad de vida de las personas (Jenaro et al., 2005; Schalock et al., 2005).

Generalmente, las evaluaciones de calidad de vida y discapacidad intelectual han puesto el relieve en la evaluación de los aspectos objetivos de la calidad de vida basados en el reporte o percepción de las personas que trabajan, educan o los asisten (Chowdhury \& Benson, 2011). Diversos son los autores como Felce y Perry (1996), Cummins (1997), Schalock (1997) y Schalock y Verdugo (2002/2003) que amplían esta perspectiva objetiva incluyendo aspectos subjetivos, que den cuenta efectiva del grado de satisfacción y preferencia desde la perspectiva de la propia persona (Verdugo et al., 2009); esto es relevante, pues numerosas investigaciones han dejado entrever que la mayo- ría de las veces existen diferencias significativas entre el carácter subjetivo, es decir, la percepción de la persona sobre su calidad de vida, y el objetivo, lo que observan sus familiares/cuidadores (Saviani-Zeoti \& Petean, 2008) o profesionales a cargo (Golubović \& Škrbić, 2013; Schmidt et al., 2010; Vega, Jenaro, Flores, Cruz \& Lerdo de Tejada, 2013).

Por lo tanto, para evaluar la calidad de vida se hace necesario conocer ambas perspectivas que permitan recolectar la información entregada por la propia persona (subjetiva) y aquella información percibida por medio de la observación directa, de la conducta del sujeto, por un tercero (objetiva), puesto que ello constituye una fuente de información para la evaluación y la provisión de servicios, ajustado a la percepción y necesidades de la persona (Verdugo, Gómez \& Arias, 2007).

En Chile, este tipo de investigaciones son escasas. Se destaca el estudio de Vega et al. (2013), que revela las diferencias entre las percepciones sobre la calidad de vida en personas institucionalizadas con discapacidad intelectual, según quienes manifiesten su opinión: profesionales a cargo, cuidadores de trato directo o directores de los centros residenciales. Otra investigación, respecto de la calidad de vida de personas con discapacidad intelectual egresados de centros de formación laboral, constató que la percepción es de carácter negativa por parte de las propias personas y de sus padres o tutores, especialmente en los aspectos referidos a la autodeterminación (Cerda, Barrera \& Díaz, 2007), lo que coincide con la investigación de Vega et al. (2012).

Por otra parte, los estudios realizados en el ámbito internacional permiten revelar que la inserción al trabajo influye significativamente, ya sea de manera directa o indirecta, en el desarrollo de las dimensiones de la calidad de vida (Alomar \& Cabré, 2005), y en el caso de las personas con discapacidad resulta más relevante, ya que — según Ibáñez y Mudarra (2005) — repercute ampliamente en su autoconcepto. Estar insertos en contextos 
laborales redunda de forma positiva en su percepción de bienestar material, autodeterminación y desarrollo personal, pues generalmente esta inserción incrementa el bienestar económico e inclusión social y así aumenta su independencia (Garvía, 2007). La formación laboral adecuada en personas con discapacidad intelectual no solo asegurará el camino hacia el empleo (Flores, Jenaro, González-Gil \& García-Calvo, 2010), sino que fomenta el desarrollo de recursos y habilidades que potencian la autonomía y la autodeterminación. Según Schalock (2010), los resultados de las personas con discapacidad intelectual mejorarán si se ajusta a su entorno y, a su vez, si la evaluación y el seguimiento se encuentran ligados (Domínguez, 2015).

En Chile, la formación laboral de los personas con discapacidad se realiza desde tres tipos paradigmas educativos, según un estudio realizado por la Consultora en Estudios, Asesorías y Planificación en Desarrollo Local (CEAS, 2002). El primero de ellos, el paradigma clínico, es un modelo basado en un enfoque no vinculado con el entorno dirigido hacia las acciones rehabilitadoras dentro del establecimiento, con un claro enfoque médico; el segundo, un paradigma social, que se vincula con las acciones específicas de formación laboral con el entorno, a través de las prácticas de los oficios en los que son preparados, y un tercer modelo, denominado en transición, el cual implica que se encuentra en proceso intermedio de migrar desde lo clínico a lo social. De la misma forma, en este estudio se postula considerar la variable género como referente en la percepción tanto subjetiva como objetiva de la calidad de vida, para analizar si se evidencian diferencias significativas en la población chilena, dado que en el resto del mundo no se ha constatado la existencia de diferencias significativas, en función del género (Mirón, Alonso, Serrano \& Sáenz, 2008; Santamaría, Verdugo, Orgaz, Gómez \& Jordán de Urríes, 2012).

Los objetivos de esta investigación buscan determinar la relación entre la formación laboral que se les brinda a personas con discapacidad intelec- tual y la calidad de vida que presentan, en función de las dimensiones propuestas por Schalock y Verdugo (2002/2003) y comparar los niveles observados tanto en las evaluaciones objetiva y subjetiva y como en sus respectivas dimensiones en función al paradigma educativo y género.

\section{Método}

\section{Participantes}

Participó un total de 751 personas con discapacidad intelectual que asisten a centros de formación laboral de la región del Bío-Bío. Además, un total de 82 profesionales a cargo de tutorizar y formar a dichas personas (directores, jefes técnicos, profesores o monitores). Las personas evaluadas asisten a alguno de los 34 establecimientos de un total de 40 , invitados a participar de la investigación. Esto corresponde al $72.7 \%$ de los establecimientos con formación laboral de personas con discapacidad intelectual de la región. En estos se consideró a la totalidad de los personas con las características mencionadas. De este grupo de personas, 293 son de género femenino (39.01\%) y 458 son de género masculino (60.99\%). Con una edad comprendida entre los 14 y los 55 años $(M=22.87 ; D T=5.86)$.

\section{Instrumento}

La investigación utilizó la Escala Integral, compuesta por dos evaluaciones: objetiva y subjetiva de la calidad de vida de personas con discapacidad intelectual (Verdugo et al., 2009). Este instrumento mide la calidad de vida, y específicamente la evaluación objetiva presenta 23 ítems que se responden con sí con no, es decir, 0 o 1 punto, con preguntas objetivas y observables, referidas a 5 dimensiones: bienestar laboral, bienestar material, bienestar emocional y físico, autodeterminación e inclusión social. La evaluación subjetiva suma la dimensión de bienestar familiar a las anteriores, y se encuentra formada por 39 ítems tipo Likert de 1 a 4 puntos, 
ante lo cual se responde según la alternativa que lo representa de mejor forma frente al enunciado planteado: "totalmente en desacuerdo" (1 punto), "en desacuerdo" ( 2 puntos), "de acuerdo" (3 puntos), "totalmente de acuerdo" (4 puntos).

\section{Procedimiento}

La aplicación de la Escala Integral Evaluación Objetiva y Subjetiva de Calidad de Vida para personas con discapacidad intelectual se realizó sobre la base del consentimiento informado de los padres de las personas, de los profesionales a cargo y de las propias personas. Se procuró crear un ambiente de confianza para su desarrollo y, además, que contarán con habilidades expresivas y comprensivas y que los profesionales a cargo tuvieran con ellos un contacto superior a tres meses.

\section{Resultados}

Los análisis estadísticos que se presentan están asociados, sobre todo, a registrar medidas de tendencia central y variabilidad, comparación de medias y análisis de fiabilidad de las escalas, mediante el coeficiente alfa de Cronbach. Se realizaron análisis de varianza simple (Anova) y el test de homogeneidad de Levene, para determinar si las varianzas dentro de los grupos de comparación poseían homocedasticidad. Cuando se encontró heterogeneidad entre las varianzas, se aplicó la prueba robusta de Welch, y el test post hoc de la diferencia significativa de Tukey, para un 99\% de confianza. En todos ellos se utilizó el programa SSPS versión 19 y también el paquete estadístico Factor 9.2 (Lorenzo-Seva \& Ferrando, 2006).

El instrumento presenta un adecuado nivel de fiabilidad general en las dos evaluaciones (objetiva y subjetiva) que la componen, pues al calcular los coeficientes alfa de Cronbach y omega de McDonald (McDonald, 1999), se obtiene para la evaluación objetiva un $\alpha=.830$ y un $\Omega=.85$. Entre tanto, para la evaluación subjetiva se obtiene un $\alpha=.827$ y un $\Omega=.92$. Se calculó el coeficiente omega de McDonald, pues es especialmente indicado para variables de naturaleza categórica u ordinal. Las diferentes dimensiones también presentan medidas adecuadas que pueden observarse en la tabla 1 .

Tabla 1

Fiabilidad de las dimensiones de las escalas objetiva y subjetiva

\begin{tabular}{lcccc}
\hline \multirow{2}{*}{ Dimensiones } & \multicolumn{2}{c}{ Escala objetiva } & \multicolumn{2}{c}{ Escala subjetiva } \\
\cline { 2 - 5 } & $\alpha$ & $\Omega$ & $\alpha$ & $\Omega$ \\
\hline Autodeterminación & .73 & .74 & .69 & .73 \\
Inclusión social & .82 & .87 & .64 & .69 \\
Bienestar laboral & .84 & .85 & .69 & .71 \\
Bienestar material & .81 & .83 & .79 & .79 \\
Bienestar emocional & .82 & .82 & .81 & .81 \\
y físico & & & & .84 \\
Bienestar familiar & & & & \\
\hline Calidad de vida: resultados & & &
\end{tabular}

Respecto de la distribución de puntuaciones medias de las ambas evaluaciones, se tiene lo siguiente: para la evaluación objetiva, la puntuación media es de 16.43 puntos, con una desviación típica de 4.59 puntos, con una asimetría negativa. La mediana es de 17 puntos, con una puntuación mínima de 0 puntos y una puntuación máxima de 23 puntos. Al observar la media de rangos percentiles, se observa que esta se encuentra en torno a 49. En el caso de la evaluación subjetiva, la puntuación media es de 132.66 puntos, con una desviación típica de 13.01 puntos, y presenta una leve asimetría negativa. La mediana es de 135 puntos, la puntuación mínima es de 77 puntos y la puntuación máxima de 156 puntos.

$\mathrm{Al}$ analizar las puntuaciones de las dimensiones de cada evaluación (objetiva y subjetiva), resumidas en la tabla 2, se observa que la dimensión con mayor nivel de consecución, considerando la proporción entre la puntuación media respecto de la puntuación máxima, para la evaluación objetiva es la dimensión de inclusión social, y es la de 
Tabla 2

Datos estadísticos de las escalas objetiva y subjetiva y sus dimensiones

\begin{tabular}{lcccccccc}
\hline \multirow{2}{*}{\multicolumn{1}{c}{ Dimensiones }} & \multicolumn{3}{c}{ Escala objetiva } & \multicolumn{5}{c}{ Escala subjetiva } \\
\cline { 2 - 8 } & Media & D. $T$. & Mín. & Máx. & Media & D. T. & Mín. & Máx. \\
\hline Autodeterminación & 4.85 & 2.26 & 0 & 8 & 24.72 & 4.43 & 8 & 32 \\
Inclusión social & 2.54 & .80 & 0 & 3 & 25.86 & 3.96 & 8 & 32 \\
Bienestar laboral & 2.46 & .84 & 0 & 3 & 14.76 & 1.81 & 6 & 16 \\
Bienestar material & 2.43 & 1.37 & 0 & 4 & 31.99 & 3.71 & 18 & 36 \\
Bienestar emocional y físico & 4.15 & 1.25 & 0 & 5 & 24.76 & 3.65 & 7 & 28 \\
Bienestar familiar & & & & & 10.56 & 1.99 & 3 & 12 \\
\hline
\end{tabular}

Nota: puntuaciones medias, desviaciones típicas y máximos y mínimos de las escalas objetiva y subjetiva y sus dimensiones.

bienestar laboral en la subjetiva. Por otra parte, la dimensión más baja en ambas evaluaciones es autodeterminación.

\section{Calidad de vida en función del género de los jóvenes}

En cuanto a puntuación global, no se constatan diferencias estadísticamente significativas, a pesar de que en cada una de las evaluaciones (objetiva y subjetiva) las puntuaciones medias del grupo masculino son levemente superiores a las del femenino. Esta situación se reitera en casi la totalidad de las dimensiones de la evaluación objetiva, salvo en las dimensiones de inclusión social y de bienestar laboral, que corresponden a la percepción de los profesionales responsables. En la dimensión inclusión social, la media de la percepción acerca del grupo masculino supera a la media del grupo femenino $(M=2.60 ; D T=.760$, y $M=2.45 ; D T=.861$, respectivamente). Estas diferencias son estadísticamente significativas, aun cuando la prueba de Levene no permitió asegurar la igualdad de varianzas, razón por la cual se aplicó la prueba de Welch $(F[1565.954]=6.026 ; p<.01)$, el tamaño del efecto calculado es $d=.234$. En la dimensión bienestar laboral, sucede una situación inversa, pues la media de la percepción acerca de las personas del grupo femenino supera a la media del grupo masculino $(M=2.56 ; D T=.773$ y $M$ $=2.39 ; D T=.877$, respectivamente). Estas diferencias son estadísticamente significativas, pero tampoco fue posible asegurar la homogeneidad de las varianzas mediante la prueba de Levene, y se aplicó la prueba de Welch $(F[1677.454]=7.177 ; p$ $<.008$ ); el tamaño del efecto calculado es $d=.242$.

En la evaluación subjetiva solo se observaron diferencias significativas en la dimensión de bienestar emocional y físico, a favor del grupo masculino $(M=24.02 ; D T=4.132$ y $M=25.23$; $D T=3.226$, respectivamente). Estas diferencias son estadísticamente significativas, aun cuando la prueba de Levene no permitió asegurar la igualdad de varianzas, razón por la cual se aplicó la prueba de Welch $(F[1514.260]=18.00 ; p<.001)$; el tamaño del efecto calculado es $d=.09$. También se constataron diferencias significativas en la dimensión bienestar material a favor del grupo femenino $(M=10.71 ; D T=1.837$ y $M=10.33 ; D T$ $=2.210$, respectivamente). Estas diferencias son estadísticamente significativas, y dado que no fue posible asegurar la homogeneidad de las varianzas mediante la prueba de Levene, se aplicó la prueba de Welch $(F[1539.827]=5.998 ; p<.01)$; el tamaño del efecto calculado es $d=.09$. 


\section{Calidad de vida en función del paradigma educativo}

$\mathrm{Al}$ analizar las puntuaciones globales medias asignadas por los profesionales en la evaluación objetiva, se observa la existencia de diferencias significativas en virtud del tipo de paradigma del centro en el que se desempeñan, es decir, paradigma clínico, social y en transición, respectivamente $(M$ $=15.58 ; D T=4.420 ; M=17.04 ; D T=4.607$ y $M$ $=15.18 ; D T=4.477)[F(2.748)=10.153 ; p<.01]$. Dichas diferencias se dan a favor de los profesionales que tienen a cargo personas que asisten a establecimientos bajo el paradigma social, respecto de aquellos que asisten a establecimientos bajo el paradigma clínico, pero no entre los otros pares de comparación (Tukey $p<.05$ ). El tamaño del efecto para la diferencia entre las medias del paradigma social respecto del paradigma clínico es $d=.07, \mathrm{y}$ entre el paradigma social y denominado en transición es $d=.09$. Estas diferencias de percepción de los profesionales se repiten en la dimensión autodeterminación, que es más alta respecto de las personas que se forman bajo el paradigma social, al compararlos con aquellas de los paradigmas clínico y en transición $(M=5.23 ; D T=2.216 ; M=4.31 ; D T$ $=2.203$ y $M=4.15 ; D T=2.334$, respectivamente) $[F(2,748)=16.342 ; p<.000]$; pero no entre este último par (Tukey $p<.05)$. El tamaño del efecto para la diferencia entre las medias del paradigma social respecto del paradigma clínico es $d=.18$, $\mathrm{y}$ entre el paradigma social y el denominado en transición es $d=.22$.

También se constatan diferencias significativas en la dimensión bienestar material $(M=2.23 ; D T$ $=1.350 ; M=2.57 ; D T=1.372$ y $M=2.10 ; D T=$ 1.294, paradigmas clínico, social y en transición, respectivamente) $[F(2,748)=6.184 ; p<.01]$, aunque solo hay una diferencia al comparar las percepciones de profesionales del paradigma social respecto del clínico; pero no entre los otros pares de comparación. El tamaño del efecto calculado es $d=.18$. Por último, en la evaluación objetiva se observa una diferencia estadísticamente significativa en la dimensión inclusión social $(M=2.42$; $D T=.871 ; M=2.64 ; D T=.741$ y $M=2.23 ; D T$ $=.842$, paradigmas clínico, social y en transición, respectivamente), coeficiente Welch ( $F$ [2101.917] $=9.176 ; p<.01)$. Esta diferencia significativa se presenta únicamente entre las percepciones de los profesionales a cargo del paradigma social respecto del clínico y en transición, pero no entre estos últimos (Tukey $p<.05$ ). El tamaño del efecto para la diferencia entre las medias del paradigma social respecto del paradigma clínico es $d=.35$, y entre el paradigma social y denominado en transición es $d=.73$.

En la evaluación subjetiva, en general, se constatan diferencias significativas respecto de las autopercepciones de las personas en función del paradigma bajo el cual se forman: clínico, social y en transición $(M=134.33 ; D T=13.111 ; M=$ $132.05 ; D T=12.671$ y $M=128.15 ; D T=14.719$, respectivamente) $[F(2.748)=5.069 ; p<.01]$, aunque las diferencias solo se dan entre aquellos que se forman bajo el paradigma clínico respecto de los que se forman bajo el paradigma en transición (Tukey $p<.05$ ) y el tamaño del efecto es $d=.03$. Al analizar las dimensiones, se observan diferencias significativas en bienestar emocional y físico, entre las percepciones de los estudiantes que se forman bajo el paradigma clínico, social y en transición ( $M$ $=25.27 ; D T=3.517 ; M=24.47 ; D T=3.737$ y $M=$ $24.62 ; D T=3.258$, respectivamente) $[F(2.748)=$ $4.014 ; p<.01])$ y estas diferencias son únicamente entre las percepciones de los estudiantes que se forman bajo el paradigma clínico respecto de los que se forman bajo el paradigma social (Tukey $p$ $<.05)$ y el tamaño del efecto es $d=.06$.

\section{Discusión}

Los resultados de la Escala Integral Evaluación Objetiva y Subjetiva de la Calidad de Vida de personas con discapacidad dejan en evidencia que la percepción de los profesionales chilenos a cargo de 
los personas con discapacidad intelectual da cuenta de un nivel de consecución relativamente alto (71.4\%), al comparar la puntuación media con la puntuación ideal; además, dicha percepción es muy homogénea. En cuanto a la evaluación subjetiva, se observa una mayor percepción de consecución por parte de las propias personas $(86.5 \%)$ con un alto nivel de homogeneidad en esos juicios. Ello permite inferir que las percepciones de ambos grupos informantes son favorables y congruentes en general, en los diversos aspectos y dimensiones de la calidad de vida evaluadas, lo que resulta concordante con investigaciones internacionales (Aróstegui, 2002; Gómez-Vela \& Verdugo, 2004). Del mismo modo, esta percepción favorable también se observó en una investigación realizada en España con personas con discapacidad, en la cual se aplicó la misma escala integral, y en el estudio transcultural en los países de Argentina, Brasil y Colombia se constató que todas las puntuaciones medias de las dimensiones superan la media teórica (Verdugo, Gómez, Arias \& Navas, 2012a).

La percepción de las propias personas es superior a la de los profesionales a cargo. Presumiblemente, esta diferencia radica en el hecho de que, en general, en investigaciones con poblaciones de personas con discapacidad intelectual sus miembros tienden a presentar niveles de calidad superiores al promedio de otros grupos (Mirón et al., 2008; Verdugo et al., 2012b). Al analizar las dimensiones de la evaluación objetiva y subjetiva, se constató que en la evaluación objetiva la dimensión mejor evaluada resultó ser la de inclusión social, a diferencia de los resultados de la investigación de Gómez, Verdugo, Arias, Navas y Schalock (2013), en la cual obtuvo la más baja valoración. Estos resultados positivos se pueden explicar por las nuevas políticas públicas que se han incrementado en Chile desde 2005, como la Política de Educación Especial y la Ley 20.422, respecto a normas sobre igualdad de oportunidades e de inclusión social de personas con discapacidad (Misterio de Planificación, 2010).
En la evaluación subjetiva, la dimensión en la cual las personas perciben mayor nivel de consecución es bienestar laboral, presumiblemente porque en este proceso de formación laboral sienten agrado por la especialidad de su taller laboral, además de un sentimiento de aceptación de su grupo, lo que los identifica con éste y sus compañeros, lo que coincide con los resultados de otras investigaciones (Ibáñez \& Mudarra, 2005), aun cuando esta investigación evaluó a personas con discapacidad en entornos de trabajo, donde los encuestados manifestaron una muy alta satisfacción con su entorno laboral y expresaron que les gusta el trabajo y que no hay diferencias por edad, género o tipo de discapacidad (Holburn, Cea, Coull \& Goode, 2007; Ibáñez \& Mudarra, 2005).

La dimensión que presentó menor porcentaje de consecución en ambas evaluaciones (objetiva y subjetiva) fue autodeterminación. De esta manera, se infiere que en general los profesionales chilenos perciben dificultades en las personas a su cargo para tomar decisiones de forma autónoma, hacerse responsables de sí mismos y participar de manera independiente en su medio, lo que coincide con los hallazgos de otras investigaciones, en las cuales se señala que las personas con discapacidad se perciben como menos autodeterminadas que sus iguales sin discapacidad (Santamaría et al., 2012; Wehmeyer et al., 2007). Al parecer, las familias tienden a frenar los deseos de realización de tareas cotidianas, como una manifestación clara de sobreprotección. Estos resultados en el ámbito de la autodeterminación coinciden con los hallazgos internacionales (Gómez-Vela \& Verdugo, 2004). En otra investigación llevada a cabo en Chile se observa que la situación no ha variado de forma sustantiva, en cuanto al grado de consecución en la dimensión de autodeterminación, pues en ese momento era la más baja tanto en la percepción de los padres como de los propios personas (Cerda et al., 2007).

$\mathrm{Al}$ analizar las percepciones respecto de la calidad de vida de las personas, en las puntuaciones 
globales de las perspectivas objetiva y subjetiva, en función del género, no se constata la existencia de diferencias significativas, lo que coincide con el mismo tipo de hallazgo en otras investigaciones (Mirón et al., 2008; Santamaría et al., 2012; Verdugo et al., 2012a). Al realizar un examen más específico dentro de la evaluación objetiva, se constata la existencia de diferencias en la dimensión inclusión social a favor del grupo masculino, lo que coincide con los hallazgos de un estudio realizado con una muestra española realizada por Verdugo et al. (2012a); y en el caso de la dimensión bienestar laboral, la diferencia favorece al grupo femenino.

En este sentido, Meneses (2009) señala que las mujeres presentan mayores desigualdades en el plano social y que se produce un efecto aún mayor en las que presentan discapacidad intelectual, lo cual podría dar sentido a las diferencias percibidas por los profesionales a cargo en favor de los hombres respecto de las mujeres en la dimensión de inclusión social. Del mismo modo, en la evaluación subjetiva, el grupo masculino supera al grupo femenino en su nivel de calidad de vida en la dimensión de bienestar emocional y físico, lo que coincide con los resultados encontrados por Gómez-Vela y Verdugo (2004), Meneses (2009) y Verdugo et al. (2012a), quienes señalan que los hombres con discapacidad presentan percepciones más altas en lo que respecta a salud que las mujeres.

Por otra parte, al comparar la percepción sobre la calidad de vida de las personas que asisten a centros de formación laboral, desde la perspectiva de las propias personas (evaluación subjetiva) o desde la perspectiva de los profesionales a cargo (evaluación objetiva) en función del paradigma que guía la actividad de formación en ellos, se constata que las personas que se forman bajo el paradigma social tienen una percepción más favorable en la dimensión de autodeterminación. Esta evidencia podría encontrar su explicación en que el modelo social basa sus actividades con la comunidad y ello facilita el empleo con apoyo, y es una metodología muy utilizada en personas con discapacidad intelectual para promover su integración sociolaboral, pues se fomentan acciones que se vinculan plenamente en el entorno (CEAS, 2002). Además, se proveen y entregan apoyos tanto dentro como fuera de su vida laboral (Jordán de Urríes, 2008). Esto es avalado por Vega (2007), que señala que los planteamientos tradicionales, centrados en la asistencia y en la recuperación de las capacidades funcionales, tienen que ser sustituidos por otros que destaquen mucho más la identificación y la eliminación de los diversos obstáculos a la igualdad de oportunidades y a la plena participación de las personas con discapacidad en todos los aspectos de la vida.

De acuerdo con los resultados obtenidos en este estudio, se concluye que la Escala Integral Evaluación Objetiva y Subjetiva de la Calidad de Vida para personas con discapacidad intelectual es un instrumento adecuado para la evaluación de personas con discapacidad intelectual, dado que posee evidencia de validez y constructo teórico comprobado (Gómez, Arias, Verdugo \& Navas, 2012).

Los resultados obtenidos en cada una de las evaluaciones tanto objetivas como subjetivas, especialmente en la dimensión de autodeterminación, insta a los profesionales que trabajan en centros de formación laboral a promover y potenciar aspectos referidos a habilidades que fomenten la autodeterminación (Wehmeyer, 2009). De este modo, que se fundamente la toma de decisiones en el trabajo con personas con discapacidad intelectual con un enfoque basado en la persona y se entreguen adecuados programas de formación que permitan generar su independencia económica y posibiliten su inclusión social.

Esta investigación presenta algunas limitaciones que es necesario considerar para otros estudios, como el realizar una evaluación objetiva que no solo contemple la percepción de los profesionales o tutores que trabajan con los personas con discapacidad, sino también la de sus familias, pues son estas la primera fuente de información, la más cercana y confiable (Vega et al., 2012). 


\section{Referencias}

Alomar, E. \& Cabré, M. (2005). El trabajo de personas con discapacidad intelectual en entorno normalizado. Síndrome de Down, 22, 118-124. Recuperado de http://www.downcantabria.com/ revistapdf/87/118-124.pdf

Aróstegui, I. (2002). Calidad de vida y retraso mental. Bilbao: Mensajero.

Cerda, G., Barrera, D. \& Díaz, C. (2007). Calidad de vida de personas con discapacidad intelectual de la provincia de Concepción. Paideia, 47,149-165.

Chowdhury, M. \& Benson, B. (2011). Deinstitutionalization and quality of life of individuals with intellectual disability: A review of the international literature. Journal of Policy and Practice in Intellectual Disabilities, 8(4), 256-265. doi:10.1111/j.17411130.2011.00325.x

Consultora en Estudios, Asesorías y Planificación en Desarrollo Local. (2002). Situación de la formación laboral en educación especial. Recuperado de http://www.ongentorno.cl/material/Publicaciones/Educacion/Formacion\%20Laboral\%20 en $\% 20$ Educacion $\% 20$ Especial $\% 20$ CEAS $\% 20$ LTDA1.pdf

Cummins, R. (1997). Bases para el desarrollo de un modelo de evaluación para personas con discapacidad intelectual adultas que viven en servicios residenciales. Salamanca: INICO. Recuperado de http://sid.usal.es/idocs/F8/FDO25938/Bases modelo_de_evaluacixn_CdV_y_Apoyos.pdf

Domínguez, S. (2015). Propiedades psicométricas de una escala de calidad de vida para personas adultas con discapacidad intelectual. Psychologa: Avances de la Disciplina, 9(1), 29-43.

Felce, D. \& Perry, J. (1996). Bases para el desarrollo de un modelo de evaluación para personas con discapacidad intelectual adultas que viven en servicios residenciales. Salamanca: INICO. Recuperado de http://sid.usal.es/idocs/F8/ FDO25938/Bases_modelo_de_evaluacixn_CdV_y_Apoyos.pdf
Flores, N., Jenaro, C., González-Gil, F., \& García-Calvo, P. (2010). Análisis de la calidad de vida laboral en trabajadores con discapacidad. Zerbitzuan, 47, 95-107.

Garvía, B. (2007). Para llegar a ser adulto. Revista Médica Internacional sobre el Sindrome de Down, 11(2), 30-32. doi: 10.1016/S11382074(07)70036-2

Golubović, S., \& Škrbić, R. (2013). Agreement in quality of life assessment between adolescents with intellectual disability and their parents. Research in Developmental Disabilities, 34(6), 1863-1869. doi: 10.1016/j.ridd.2013.03.006

Gómez, L., Arias, B., Verdugo, M., \& Navas, P. (2012). Application of the Rasch Rating Scale Model to the assessment of quality of life of persons with intellectual disability. Journal of Intellectual \& Developmental Disability, 37(29), 141-150. doi: 10.3109/13668250.2012.682647

Gómez, L., Verdugo, M., \& Arias, B. (2010). Calidad de vida individual: avances en su conceptualización y retos emergentes en el ámbito de la discapacidad. Behavioral Psychology, 18(3), 453-472. Recuperado de http://www. thefreelibrary.com/Calidad+de+vida + individual $\% 3 \mathrm{~A}+$ avances + en + su + conceptualizacion $+\mathrm{y}+$ retos...-a0314254335

Gómez, L., Verdugo, M., Arias, B., \& Arias, V. (2010). A comparison of alternative models of individual quality of life for social service recipients. Social Indicators Research. 101, 109-126. doi: 10.1007/s11205-010-9639-y

Gómez, L., Verdugo, M., Arias, B., Navas P. \& Schalock, R. (2013). The development and use of provider profiles at the organizational and systems level. Evaluation and Program Planning, 40, 17-26. doi.org/10.1016/j.evalprogplan.2013.05.001

Gómez-Vela, M. \& Verdugo, M. (2004). El cuestionario de evaluación de la calidad de vida de personas de educación secundaria obligatoria: descripción, validación inicial y resultados obtenidos tras su aplicación en una muestra de 
adolescentes con discapacidad y sin ella. Siglo Cero (212), 5-17. Recuperado de http://www. feaps.org/archivo/publicaciones-feaps/siglo-cero/numeros-gratuitos/292-n212.html

Henao, C. \& Gil, L. (2009). Calidad de vida y situación de discapacidad. Hacia la Promoción de la Salud, 4(2), 112-125. Recuperado de http:// promocionsalud.ucaldas.edu.co/downloads/ PromocionSalud14\%282\%29_9.pdf

Holburn, S., Cea, C., Coull, L., \& Goode, D. (2007). What is working and not working: using focus groups to address quality of life of people living in group homes. Journal of Developmental and Physical, 20, 1-9. doi: 10.1007/s10882-0079074-4

Ibáñez, P. \& Mudarra, M. (2005). Relaciones sociales en el empleo, en trabajadores con discapacidad. Educación XXI (8), 105-125. Recuperado de http://e-spacio.uned.es/revistasuned/index.php/ educacionXX1/article/viewFile/345/298

Jenaro, C., Verdugo, M., Caballo, C., Balboni, G., Lachapelle, Y., Otrebski, W. \& Schalock, R. (2005). Cross-cultural study of person-centered quality of life domains and indicators: A replication. Journal of Intellectual Disability Research, 49, 734-739. doi: 10.111/j.13652788.2005.00742x

Jordán de Urríes, F. (2008). Empleo con apoyo para personas con enfermedad mental. Intervención Psicosocial, 17, 299-305. Recuperado de http://www.redalyc.org/articulo. oa? id $=179814023006$

Ministerio de Planificación. (2010). Ley 20.422: Establece normas sobre igualdad de oportunidades e inclusión social de personas con discapacidad. Biblioteca Congreso Nacional, Chile. Recuperado de http://www.leychile.cl/ Navegar?idNorma $=1010903 \&$ idParte $=$ \&idVersion $=2013-01-01$

Lorenzo-Seva, U. \& Ferrando, P. (2006). Factor: A computer program to fit the exploratory factor analysis model. Journal Behavior Research Me- thods, 38(1), 88-91. Recuperado de http://link. springer.com/article/10.3758\%2FBF03192753

McDonald, R. (1999). Test theory: A unified treatment. Mahwah, NJ: Lawrence Erlbaum.

Meneses, C. (2009). Género, desigualdad e inclusión Madrid: Séptimo Catálogo Español de Buenas Prácticas. Recuperado de http://habitat.aq.upm. es/boletin/n41/acmen.html

Mirón, J., Alonso, M., Serrano, A., \& Sáenz, M. (2008). Calidad de vida relacionada con la salud de personas con discapacidad intelectual en España. Revista Panamericana de Salud Pública, 21(5), 336-444. Recuperado de http://www. scielosp.org/pdf/rpsp/v24n5/06.pdf

Santamaría, M., Verdugo, M., Orgaz, B., Gómez, L., \& Jordán de Urríes, F. (2012). Calidad de vida percibida por trabajadores con discapacidad intelectual en empleo ordinario. Siglo Cero, 43(2), 46-61. Recuperado de http://sid.usal.es/idocs/ F8/ART19581/santamaria_calidadevida.pdf

Saviani-Zeoti, F. \& Petean, E. (2008). Quality of life of people with mild intellectual disability. Psicologia: Teoria e Pesquisa, 24(3), 305-311. doi:10.1590/S0102-37722008000300006

Schalock, R. (marzo de 1997). Evaluación de programas sociales: para conseguir rendimientos organizacionales y resultados personales. En Verdugo (Presidencia), II Jornadas cientificas de Investigación sobre personas con discapacidad, Salamanca, España.

Schalock, R. (abril de 2010). Aplicaciones del paradigma de calidad de vida a las personas con discapacidad intelectual y del desarrollo. En Verdugo (Presidencia), VII Seminario de Actualización Metodológica en Investigación sobre discapacidad SAID. Colección de Actas 6/2010, Universidad de Salamanca, Salamanca, España. Schalock, R. \& Verdugo, M. (2002/2003). Quality of life for human service practitioners. Washington, DC: American Association of Mental Retardation.

Schalock, R., Verdugo, M., Jenaro, C., Wang, M., Wehmeyer, M., Jiancheng, X., \& Lachapelle, 
Y. (2005). Cross-cultural study of quality of life indicators. American Journal on Mental Retardation, 110, 298-311. doi: 10.1352/0895-8017(2005)110[298:CSOQOL]2.0.CO;2

Schmidt, S., Power, M., Green, A., Lucas-Carrasco, R., Eser, E., Dragomirecka, E. \& Fleck, M. (2010). Self and proxy rating of quality of life in adults with intellectual disabilities: Results from the DISQOL study. Research in Developmental Disabilities, 31(5), 1015-1026. doi:10.1016/j. ridd.2010.04.013

Vega, A. (2007). De la dependencia a la autonomía: ¿dónde queda la educación? Educación XX1,10, 239-264. Recuperado de http://www.redalyc. org/articulo.oa? $\mathrm{id}=70601013$

Vega, V., Jenaro, C., Flores, N., Cruz, M., \& Lerdo de Tejada, A. (2012). Necesidades de apoyos de adultos con discapacidad intelectual institucionalizados en Chile, desde la perspectiva de los proveedores de servicios. Avances en Psicología Latinoamericana, 30(1), 137-145. Recuperado de: http://revistas.urosario.edu.co/index.php/ apl/article/view/1568/1838

Vega, V., Jenaro, C., Flores, N., Cruz, M. \& Lerdo de Tejada, A. (2013). Evaluación de la calidad de vida de adultos con discapacidad intelectual institucionalizados en Chile. Universitas Psychologica, 12(2), 471-481. doi:10.11144/ Javeriana.UPSY12-2.ecva

Verdugo, M., Gómez, L., Arias, B., \& Navas, P. (2012a). Evidencias de validez del modelo de calidad de vida de ocho dimensiones y aplicación de la escala integral en distintos países. En M. Verdugo, R. Canal, C. Jenaro, M. Badia \& A. Aguado, Aplicación del paradigma de calidad de vida a la intervención con personas con discapacidad desde una perspectiva integral

Fecha de recepción: 19 de mayo de 2014 fecha de aceptación: 9 de junio de 2015 (pp. 11-26). Salamanca: INICO. Recuperado de http://sid.usal.es/idocs/F8/FDO26212/Investigacion7.pdf

Verdugo M., Gómez, L., \& Arias, B. (2007). La escala integral de calidad de vida desarrollo y su estudio preliminar de sus propiedades psicométricas. Siglo Cero, 38(4), 37-56. Recuperado de http://riberdis.cedd.net/handle/11181/3170

Verdugo, M., Gómez, L., Arias, B., \& Schalock, R. (2009). Escala Integral de Calidad de Vida. Madrid: CEPE.

Verdugo, M. \& Schalock, R. (2009). Quality of life: from concept to application in the field of intellectual disabilities. Journal of Policy and Practice in Intellectual Disabilities, 6(1), 62-64. doi:10.1111/j.1741-1130.2008.00201.x

Verdugo, M., Benito, M., Orgaz, B., Jordán de Urríes, F., Martín, R., \& Santamaría, M. (2012b). Influencia del programa empleo con apoyo en la calidad de vida percibida en sus usuarios. Siglo Cero, 43(3), 243. Recuperado de http://sid.usal. es/idocs/F8/ART19585/siglo cero eca.pdf

Wang, M., Schalock, R., Verdugo, M., \& Jenaro, C. (2010). Examining the factor structure and hierarchical nature of the quality of life construct. American Journal on Intellectual and Developmental Disabilities, 115, 218-233. doi: 10.1352/1944-7558-115.3.218

Wehmeyer, M. (2009). Autodeterminación y la tercera generación de prácticas de inclusión. Revista de Educación, 349, 45-67. Recuperado de http://www.uam.es/personal_pdi/stmaria/sarrio/DOCUMENTOS,\%20ARTICULOS, \%20 PONENECIAS, $/$ re349.pdf\#page $=43$

Wehmeyer, M., Agran, M., Hughes, C., Martin, J., Mithaug, D., \& Palmer, S. (2007). Promoting self-determination in students with intellectual and developmental disabilities. En Discapacidad e inclusión manual para la docencia. Amaru: Salamanca. 